\title{
On Supersymmetry of the Covariant 3-Algebra Model for M-Theory
}

\author{
Matsuo Sato \\ Department of Natural Science, Faculty of Education, Hirosaki University, Hirosaki, Japan \\ Email: msato@cc.hirosaki-u.ac.jp
}

Received September 5, 2012; revised October 4, 2012; accepted October 12, 2012

\begin{abstract}
We examine a natural supersymmetric extension of the bosonic covariant 3-algebra model for M-theory proposed in [1]. It possesses manifest $\mathrm{SO}(1,10)$ symmetry and is constructed based on the Lorentzian Lie 3-algebra associated with the $U(N)$ Lie algebra. There is no ghost related to the Lorentzian signature in this model. It is invariant under 64 supersymmetry transformations although the supersymmetry algebra does not close. From the model, we derive the BFSS matrix theory and the IIB matrix model in a large $\mathrm{N}$ limit by taking appropriate vacua.
\end{abstract}

Keywords: M-Theory; 3-Algebra; Matrix Model; String Theory

\section{Introduction}

The BFSS matrix theory is conjectured to describe infinite momentum frame (IMF) limit of M-theory in [2] and many evidences were found. However, because of the limit, $\mathrm{SO}(1,10)$ symmetry is not manifest in these models; it includes only time and nine matrices corresponding to nine spatial coordinates. As a result, it is very difficult to derive full dynamics of M-theory. For example, we do not know the manner to describe longitudinal momentum transfer of D0-branes. Therefore, we need a covariant matrix model for M-theory that possesses manifest SO $(1,10)$ symmetry.

Recently, structures of 3-algebras [3-5] were found in the effective actions of the multiple M2-branes [6-14] ${ }^{1}$ and 3-algebras have been intensively studied [15-31]. One can expect that structures of 3-algebras play more fundamental roles in M-theory ${ }^{2}$ than the accidental structures in the effective descriptions.

The BFSS matrix theory and the IIB matrix model [35] can be obtained by the matrix regularization of the Poisson brackets of the light-cone membrane theory [36] and of Green-Schwarz string theory in Schild gauge [35], respectively. Because the regularization replaces a twodimensional integral over a world volume by a trace over matrices, the BFSS matrix theory and the IIB matrix model are one-dimensional and zero-dimensional field theories, respectively. On the other hand, the bosonic part of the membrane action has a structures of a

\footnotetext{
${ }^{1} \mathrm{ABJM}$ theory can also be rewritten in a 3-algebra manifest form [14]. ${ }^{2} \mathrm{~A}$ formulation of M-theory by a cubic matrix action was proposed by Smolin [32-34].
}

3-algebra. That is, it can be written in the 3-algebra manifest form as

$$
S=T_{M 2} \int \mathrm{d}^{3} \sigma \sqrt{g}\left(-\frac{1}{12}\left(\frac{1}{\sqrt{g}}\left\{X^{L}, X^{M}, X^{N}\right\}\right)^{2}+\Lambda\right)
$$

where $\{,$,$\} denotes Nambu-Poisson bracket [15,16]$. Therefore, a bosonic covariant 3-algebra model for Mtheory was proposed in [1].

In this paper, we examine a natural supersymmetric extension of the bosonic covariant model in $[1]^{3}$,

$$
\begin{aligned}
S & =-\frac{1}{12}\left\langle\left[X^{L}, X^{M}, X^{N}\right]^{2}\right\rangle \\
& +\frac{1}{4}\left\langle\bar{\Psi} \Gamma_{M N}\left[X^{M}, X^{N}, \Psi\right]\right\rangle .
\end{aligned}
$$

The bosons $X^{L}$ and the Majorana fermions $\Psi$ are spanned by the elements of the Lorentzian Lie 3-algebra associated with the $U(N)$ Lie algebra. This action defines a zero-dimensional field theory and possesses manifest $\mathrm{SO}(1,10)$ symmetry. By expanding fields around appropriate vacua, we derive the BFSS matrix theory and the IIB matrix model in a large $N$ limit.

\section{A Supersymmetric Extension}

We examine a following model,

$$
\begin{aligned}
S & =-\frac{1}{12}\left\langle\left[X^{L}, X^{M}, X^{N}\right]^{2}\right\rangle \\
& +\frac{1}{4}\left\langle\bar{\Psi} \Gamma_{M N}\left[X^{M}, X^{N}, \Psi\right]\right\rangle .
\end{aligned}
$$

${ }^{3}$ This extension was originally proposed in Appendix of [1]. 
where $X^{L}$ with $L=0,1, \cdots, 10$ are vectors and $\Psi$ are Majorana spinors of $\mathrm{SO}(1,10)$. This action defines a zero-dimensional field theory and possesses manifest SO $(1,10)$ symmetry. There is no coupling constant.

$X^{M}$ and $\Psi$ are spanned by the elements of the Lorentzian Lie 3-algebra associated with the $U(N)$ Lie algebra,

$$
\begin{aligned}
& X^{M}=X_{-1}^{M} T^{-1}+X_{0}^{M} T^{0}+X_{i}^{M} T^{i}, \\
& \Psi=\Psi_{-1} T^{-1}+\Psi_{0} T^{0}+\Psi_{i} T^{i},
\end{aligned}
$$

where $i=1,2, \cdots, N^{2}$. The algebra is defined by

$$
\begin{aligned}
& {\left[T^{-1}, T^{a}, T^{b}\right]=0,} \\
& {\left[T^{0}, T^{i}, T^{j}\right]=\left[T^{i}, T^{j}\right]=f_{k}^{i j} T^{k},} \\
& {\left[T^{i}, T^{j}, T^{k}\right]=f^{i j k} T^{-1},}
\end{aligned}
$$

where $a, b=-1,0,1,2, \cdots, N^{2}$ and $f^{i j k}=f_{l}^{i j} h^{l k}$ is totally anti-symmetrized. $\left[T^{i}, T^{j}\right]$ is a Lie bracket of the $\mathrm{U}(\mathrm{N})$ Lie algebra. The metric of the elements is defined by

$$
\begin{aligned}
& \left\langle T^{-1}, T^{-1}\right\rangle=0,\left\langle T^{-1}, T^{0}\right\rangle=-1, \\
& \left\langle T^{-1}, T^{i}\right\rangle=0,\left\langle T^{0}, T^{0}\right\rangle=0, \\
& \left\langle T^{0}, T^{i}\right\rangle=0, \quad\left\langle T^{i}, T^{j}\right\rangle=h^{i j} .
\end{aligned}
$$

By using these relations, the action is rewritten as

$$
\begin{aligned}
S & =\operatorname{tr}\left(-\frac{1}{4}\left(X_{0}^{L}\right)^{2}\left[X_{M}, X_{N}\right]^{2}+\frac{1}{2}\left(X_{0}^{M}\left[X_{M}, X_{N}\right]\right)^{2}\right. \\
& +\frac{1}{2} X_{0}^{M} \bar{\Psi} \Gamma_{M N}\left[X^{N}, \Psi\right] \\
& \left.-\frac{1}{2} \bar{\Psi}_{0} \Gamma_{M N} \Psi\left[X^{M}, X^{N}\right]\right),
\end{aligned}
$$

where $X^{M}=X_{i}^{M} T^{i}$ and $\Psi=\Psi_{i} T^{i}$. There is no ghost in the theory, because $X_{-1}^{M}$ or $\Psi_{-1}$ does not appear in the action ${ }^{4}$.

Let us summarize symmetry of the action. First, gauge symmetry is the $\mathrm{N}^{2}$-dimensional translation and $\mathrm{U}(\mathrm{N})$ symmetry associated with the Lorentzian Lie 3-algebra [10].

Second, there are two kinds of shift symmetry. First one is the eleven-dimensional translation symmetry generated by

$$
\delta X^{M}=\eta^{M},
$$

Where $X^{M} \in U(N), \eta^{M} \in U(1)$ and the other fields are not transformed. Second one is a part of supersymmetry, so called the kinematical supersymmetry, generated by

$$
\delta_{1} \Psi=\varepsilon_{1},
$$

${ }^{4}$ Ghost-free Lorentzian 3-algebra theories were studied in $[37,38]$ where $\Psi \in U(N), \quad \varepsilon_{1} \in U(1)$ and the other fields are not transformed.

Third, the action is invariant under another part of supersymmetry transformation, so called the dynamical supersymmetry transformation,

$$
\begin{aligned}
& \delta_{2} X^{M}=i \bar{\varepsilon}_{2} \Gamma^{M} \Psi \\
& \delta_{2} X_{0}^{M}=i \bar{\varepsilon}_{2} \Gamma^{M} \Psi_{0} \\
& \delta_{2} \Psi=-\frac{i}{2}\left[X^{L}, X^{M}\right] X_{0}^{N} \Gamma_{L M N} \varepsilon_{2} \\
& \left(\delta_{2} \bar{\Psi}_{0}\right) \tilde{\Psi}=-\delta_{0} S,
\end{aligned}
$$

where $\tilde{\Psi}=\frac{1}{2} \operatorname{tr}\left(\Gamma_{M N} \Psi\left[X^{M}, X^{N}\right]\right)$ and $\delta_{0} S$ is the variation of the action (5) under (8), (9) and (10).

We should note that the above super transformation is slightly different with a 3-algebra manifest super transformation, which is a straightforward analogue to that of the BLG theory for multiple M2-branes;

$$
\begin{aligned}
& \delta X^{M}=i \widetilde{\varepsilon}^{M} \Psi \\
& \delta \Psi=-\frac{i}{6}\left[X_{L}, X_{M}, X_{N}\right] \Gamma^{L M N} \varepsilon .
\end{aligned}
$$

If we decompose this transformation, (8), (9) and (10) are the same, but (11) is different. In the analogue case, $\delta \Psi_{0}=0$. There is no such symmetry ${ }^{5}$ because $\delta_{0} S \neq 0$.

In the Lorentzian case, the action does possess supersymmetry because $\delta_{2} \Psi_{0}$ cancels $\delta_{0} S$. However, $\delta_{2} \Psi_{0}$ is inconsistent with the 3-algebra symmetry. As a result, the supersymmetry algebra does not close, although it closes in a $X^{M}$ sector as one can see below.

The commutators among the supersymmetry transformations act on $X^{M}$ as

$$
\begin{aligned}
& \left(\delta_{1} \tilde{\delta}_{2}^{\prime}-\tilde{\delta}_{2}^{\prime} \delta_{1}\right) X^{M}=-i \overline{\varepsilon_{1}} \Gamma^{M} \varepsilon_{2}^{\prime} \\
& \left(\delta_{1} \delta_{1}^{\prime}-\delta_{1}^{\prime} \delta_{1}\right) X^{M}=0 \\
& \left(\delta_{2} \delta_{2}^{\prime}-\delta_{2}^{\prime} \delta_{2}\right) X^{M}=\Lambda_{a b}\left[T^{a}, T^{b}, X^{M}\right]_{i} T^{i},
\end{aligned}
$$

where $\Lambda_{a b}=-i \bar{\varepsilon}_{2}^{\prime} \Gamma_{L N} \varepsilon_{2} X_{a}^{L} X_{b}^{N}$.

If we change a basis of the supersymmetry transformations as

$$
\begin{aligned}
& \tilde{\delta}_{1}=\delta_{2}+\delta_{1} \\
& \tilde{\delta}_{2}=i\left(\delta_{2}-\delta_{1}\right),
\end{aligned}
$$

up to the gauge transformation, we obtain

$$
\begin{aligned}
& \left(\tilde{\delta}_{1}^{\prime} \tilde{\delta}_{1}-\tilde{\delta}_{1} \tilde{\delta}_{1}^{\prime}\right) X^{M}=\delta_{\eta} X^{M} \\
& \left(\tilde{\delta}_{2}^{\prime} \tilde{\delta}_{2}-\tilde{\delta}_{2} \tilde{\delta}_{2}^{\prime}\right) X^{M}=\delta_{\eta} X^{M} \\
& \left(\tilde{\delta}_{2}^{\prime} \tilde{\delta}_{1}-\tilde{\delta}_{1} \tilde{\delta}_{2}^{\prime}\right) X^{M}=0,
\end{aligned}
$$

\footnotetext{
${ }^{5}$ This fact was originally shown in [39].
} 
where $\delta_{\eta}$ is a translation.

These 64 supersymmetry transformations are summarised as $\Delta=\left(\tilde{\delta}_{1}, \tilde{\delta}_{2}\right)$ and (14) implies the $\mathcal{N}=2$ supersymmetry algebra in eleven dimensions in the $X^{M}$ sector,

$$
\left(\Delta \Delta^{\prime}-\Delta^{\prime} \Delta\right) X^{M}=\delta_{\eta} X^{M} .
$$

Because the low energy effective description of Mtheory is given by the $\mathcal{N}=1$ eleven-dimensional supergravity, the $\mathcal{N}=2$ supersymmetry in this sector is necessarily broken into the $\mathcal{N}=1$ supersymmetry, spontaneously. In the next section, we will show that the model reduces to the BFSS matrix theory and the IIB matrix model in a large $\mathrm{N}$ limit if appropriate vacua are chosen.

Because the commutators among the supersymmetry transformations of $X^{M}$ result in the eleven-dimensional translation (6), eigen values of $X^{M} \in U(N)$ should be interpreted as eleven-dimensional space-time ${ }^{6}$. In the next section, when we derive the BFSS matrix theory and the IIB matrix model, $X^{i}(i=1, \cdots, 9) \in U(N)$ and $X^{i}(i=0, \cdots, 9) \in U(N)$ are identified with matrices in the BFSS matrix theory and the IIB matrix model respectively. Therefore, our interpretation is consistent with the space-time interpretation in these models.

\section{BFSS Matrix Theory and IIB Matrix Model from Covariant 3-Algebra Model for M-Theory}

The covariant 3-algebra model for M-theory possesses a large moduli that includes simultaneously diagonalizable configurations. By treating appropriate configurations as backgrounds, we derive the BFSS matrix theory and the IIB matrix model in the large $N$ limit.

We consider backgrounds

$$
\begin{gathered}
\bar{X}^{\mu}=p^{\mu}=\operatorname{diag}\left(p_{1}^{\mu}, p_{2}^{\mu}, \cdots, p_{N}^{\mu},\right) \\
\bar{X}^{I}=0 \\
\bar{X}_{0}^{M}=\frac{1}{g} \delta_{10}^{M}, \\
\bar{\Psi}=\bar{\Psi}_{0}=0,
\end{gathered}
$$

where $\mu=0,1, \cdots, d-1(d \leq 10)$ and $I=d, \cdots, 10$. $\left(p_{0}^{i}, p_{1}^{i}, \cdots, p_{d-1}^{i}\right) \quad(i=1, \cdots, N)$ represent $N$ points randomly distributed in a d-dimensional space. There are infinitely many such configurations. $X_{0}^{M}$ represents an eleven-dimensional constant vector. By using $\mathrm{SO}(1,10)$ symmetry, we can choose (3) as a background without loss of generality. $g$ will be identified with a coupling constant. $g \rightarrow \infty$ corresponds to $\bar{X}_{0}^{M}=0$, which leads

${ }^{6}$ This kind of mechanism and interpretation was originally found in [35]. to $\mathrm{SO}(1,10)$ symmetric vacua.

We assume all the backgrounds (1), (2), (3) and (4) as independent vacua and fix them in the large $\mathrm{N}$ limit [40]. Thus, we do not integrate $X_{0}^{M}, \Psi_{0}$ or the diagonal elements of $a_{\mu}$ and we expand the fields around the backgrounds as,

$$
\begin{aligned}
& X_{\mu}=p_{\mu}+a_{\mu} \\
& X_{I}=x_{I} . \\
& \Psi=\psi,
\end{aligned}
$$

where we impose a chirality condition

$$
\Gamma^{10} \psi=\psi .
$$

Under these conditions, the first term of the action (5) is rewritten as

$$
\begin{aligned}
& S_{1}=\operatorname{tr}\left(-\frac{1}{4}\left(X_{0}^{L}\right)^{2}\left[X_{M}, X_{N}\right]^{2}\right) \\
& \begin{aligned}
=-\frac{1}{4 g^{2}} \operatorname{tr} & \left(\left[p_{\mu}+a_{\mu}, p_{v}+a_{v}\right]^{2}+2\left[p_{\mu}+a_{\mu}, x^{I}\right]^{2}\right. \\
& \left.+\left[x^{I}, x^{J}\right]^{2}\right) .
\end{aligned}
\end{aligned}
$$

The second term is

$$
\begin{aligned}
S_{2} & =\frac{1}{2} \operatorname{tr}\left(\left(X_{0}^{M}\left[X_{M}, X_{N}\right]\right)^{2}\right) \\
& =\frac{1}{2 g^{2}} \operatorname{tr}\left(\left[p_{\mu}+a_{\mu}, x^{10}\right]^{2}+\left[x^{10}, x^{I}\right]^{2}\right) .
\end{aligned}
$$

As a result, the total action is independent of $x^{10}$ as follows,

$$
\begin{aligned}
S= & -\frac{1}{g^{2}} \operatorname{tr}\left(\frac{1}{4}\left[p_{\mu}+a_{\mu}, p_{v}+a_{v}\right]^{2}\right. \\
& +\frac{1}{2}\left[p_{\mu}+a_{\mu}, x^{i}\right]^{2}+\frac{1}{4}\left[x^{i}, x^{j}\right]^{2} \\
& \left.+\frac{g}{2} \bar{\psi} \Gamma^{\mu}\left[p_{\mu}+a_{\mu}, \psi\right]+\frac{g}{2} \bar{\psi} \Gamma^{i}\left[x_{i}, \psi\right]\right),
\end{aligned}
$$

where $i, j=d, \cdots, 9$. In the large $N$ limit, this action is equivalent to

$$
\begin{aligned}
S=-\frac{1}{g^{2}} \int \mathrm{d}^{d} \sigma & \operatorname{tr}\left(\frac{1}{4} F_{\mu \nu}^{2}-\frac{1}{2}\left(D_{\mu} x^{i}\right)^{2}+\frac{1}{4}\left[x^{i}, x^{j}\right]^{2}\right. \\
+ & \left.\frac{i}{2} \bar{\psi} \Gamma^{\mu} D_{\mu} \psi+\frac{1}{2} \bar{\psi} \Gamma^{i}\left[x_{i}, \psi\right]\right),
\end{aligned}
$$

where $\psi$ is redefined to $\frac{1}{\sqrt{g}} \psi$. This fact is proved perturbatively and non-perturbatively in the large $N$ limit as in the case of the large $\mathrm{N}$ reduced model [41-44].

Under the conditions (1)-(6), the super transformations (8) and (10) reduces to 


$$
\begin{aligned}
& \delta a^{\mu}=i \bar{\varepsilon} \Gamma^{\mu} \psi \\
& \delta x^{I}=i \bar{\varepsilon} \Gamma^{I} \psi \\
& \delta \psi=-\frac{i}{2 g}\left(\left[p_{\mu}+a_{\mu}, p_{v}+a_{v}\right] \Gamma^{\mu v}+\left[x^{i}, x^{j}\right] \Gamma^{i j}\right) \mathcal{E},
\end{aligned}
$$

by which (9) is invariant. Moreover, (9) and (11) reduces to

$$
\begin{aligned}
& \delta X_{0}^{M}=0 \\
& \delta \Psi_{0}=0,
\end{aligned}
$$

because the action (5) reduces to the action (9) and $\delta_{0} S=0$. This is consistent with the fact that $X_{0}^{M}$ and $\Psi_{0}$ are fixed.

Therefore, if we choose the backgrounds with $d=1$, we obtain the BFSS matrix theory in the large $N$ limit,

$$
\begin{aligned}
S & =\frac{1}{4 g^{2}} \int \mathrm{d} \tau \operatorname{tr}\left(2\left(D_{0} x^{i}\right)^{2}-\left[x^{i}, x^{j}\right]^{2}\right. \\
& \left.-\frac{i}{2} \bar{\psi} \Gamma^{0} D_{0} \psi-\frac{1}{2} \bar{\psi} \Gamma^{i}\left[x_{i}, \psi\right]\right) .
\end{aligned}
$$

If we choose those with $d=0$, we obtain the IIB matrix model in the large $N$ limit,

$$
S=-\frac{1}{4 g^{2}} \operatorname{tr}\left(\left[x^{i}, x^{j}\right]^{2}+\frac{1}{2} \bar{\psi} \Gamma^{i}\left[x_{i}, \psi\right]\right) .
$$

We also obtain matrix string theory [45-47] when $d=2$ and $A d S_{5} / C F T_{4}$ [48] when $d=4$.

\section{Conclusion and Discussion}

In this paper, we have studied a natural supersymmetric extension of the bosonic covariant 3-algebra model for M-theory proposed in [1]. It possesses manifest $\mathrm{SO}(1,10)$ symmetry. The action is invariant under 64 supersymmetry transformations, although the supersymmetry algebra does not close. In this model, the eleven-dimensional space-time is given by eigen values of the $\mathrm{U}(\mathrm{N})$ part of the bosonic fields $X^{M}$. From this action, by choosing appropriate vacua, we have derived the BFSS matrix theory and the IIB matrix model in a large $\mathrm{N}$ limit.

In order to obtain a covariant 3-algebra model for M-theory by means of a matrix regularization of a supermembrane action, the action must be written only with the Nambu brackets. Then, the action must be invariant under constant shifts of the fermions, that is under the kinematical supersymmetry transformations. The number of them is 32 because the Majorana fermions possess 32 components for covariance. Thus, the total number of the dynamical and kinematical supersymmetries exceeds the number of the $\mathcal{N}=1$ supersymmetries. Therefore, there does not exist a $\mathcal{N}=1$ supersymmetric covariant 3-algebra model for M-theory that is obtained by a matrix regularization of a supermembrane action. As a result, there are two possibilities for 3-algebra models for Mtheory. One is a covariant 3-algebra model for M-theory that possesses more than 32 supersymmetries as in this paper. Another is a $\mathcal{N}=1$ supersymmetric 3-algebra model for M-theory that is obtained by a matrix regularization of a non-covariant supermembrane action ${ }^{7}$.

\section{Acknowledgements}

We would like to thank T. Asakawa, K. Hashimoto, N. Kamiya, H. Kunitomo, T. Matsuo, S. Moriyama, K. Murakami, J. Nishimura, S. Sasa, F. Sugino, T. Tada, S. Terashima, S. Watamura, K. Yoshida, and especially H. Kawai and A. Tsuchiya for valuable discussions.

\section{REFERENCES}

[1] M. Sato, “Covariant Formulation of M-Theory,” International Journal of Modern Physics A, Vol. 24, No. 27, 2009, pp. 5019-5024. doi:10.1142/S0217751X09047661

[2] T. Banks, W. Fischler, S. H. Shenker and L. Susskind, "M Theory as a Matrix Model: A Conjecture," Physical Review D, Vol. 55, No. 8, 1997, p. 5112-5128. doi:10.1103/PhysRevD.55.5112

[3] V. T. Filippov, "N-Lie Algebras," Sbornik: Mathematics, Vol. 26, No. 6, 1985, pp. 126-140.

[4] N. Kamiya, "A Structure Theory of Freudenthal-Kantor Triple Systems,” Journal of Algebra, Vol. 110, No. 1, 1987, pp. 108-123. doi:10.1016/0021-8693(87)90038-X

[5] S. Okubo and N. Kamiya "Quasi-Classical Lie Superalgebras and Lie Supertriple Systems," Communications in Algebra, Vol. 30, No. 8, 2002, pp. 3825-3850.

[6] J. Bagger and N. Lambert, "Modeling Multiple M2's," Physical Review D, Vol. 75, 2007, Article ID: 045020, p 7. doi:10.1103/PhysRevD.75.045020

[7] A. Gustavsson, "Algebraic Structures on Parallel M2-Branes,” Nuclear Physics B, Vol. 811, No. 1-2, 2009, pp. 6676. doi:10.1016/j.nuclphysb.2008.11.014

[8] J. Bagger and N. Lambert, "Gauge Symmetry and Supersymmetry of Multiple M2-Branes," Physical Review D, Vol. 77, No. 6, 2008, Article ID: 065008, p 6. doi:10.1103/PhysRevD.77.065008

[9] S. Mukhi and C. Papageorgakis, "M2 to D2," Journal of High Energy Physics, Vol. 0805, 2008, p. 085.

[10] J. Gomis, G. Milanesi and J. G. Russo, "Bagger-Lambert Theory for General Lie Algebras,” Journal of High Energy Physics, Vol. 0806, 2008, p. 075.

[11] S. Benvenuti, D. Rodriguez-Gomez, E. Tonni and H. Verlinde, "N=8 Superconformal Gauge Theories and M2

${ }^{7}$ In [49-51], because a supermembrane action that is 3-algebra manifest but a non-covariant, is obtained by fixing only the $\mathcal{K}$-symmetry of the covariant supermembrane action, it is expected to obtain all the physical observables in M-theory by using the corresponding 3-algebra model. 
Branes,” Journal of High Energy Physics, Vol. 0901, 2009, p. 078.

[12] P.-M. Ho, Y. Imamura and Y. Matsuo, "M2 to D2 Revisited,” Journal of High Energy Physics, Vol. 0807, 2008, p. 003.

[13] O. Aharony, O. Bergman, D. L. Jafferis and J. Maldacena, "N=6 Superconformal Chern-Simons-Matter Theories, M2-Branes and Their Gravity Duals," Journal of High Energy Physics, Vol. 0810, 2008, p. 091.

[14] J. Bagger and N. Lambert, "Three-Algebras and $\mathrm{N}=6$ Chern-Simons Gauge Theories," Physical Review D, Vol. 79, No. 2, 2009, Article ID: 025002, p 8. doi:10.1103/PhysRevD.79.025002

[15] Y. Nambu, “Generalized Hamiltonian Dynamics,” Physical Review D, Vol. 7, No. 8, 1973, pp. 2405-2412. doi:10.1103/PhysRevD.7.2405

[16] H. Awata, M. Li, D. Minic and T. Yoneya, "On the Quantization of Nambu Brackets," Journal of High Energy Physics, Vol. 0102, 2001, 013. doi:10.1088/1126-6708/2001/02/013

[17] D. Minic, "M-Theory and Deformation Quantization," 2007. arXiv:hep-th/9909022

[18] J. Figueroa-O'Farrill and G. Papadopoulos, "Plueckertype Relations for Orthogonal Planes," Journal of Geometry and Physics, Vol. 49, No. 3-4, 2004, pp. 294-331. doi:10.1016/S0393-0440(03)00093-7

[19] G. Papadopoulos, "M2-Branes, 3-Lie Algebras and Plucker Relations," Journal of High Energy Physics, Vol. 0805, 2008, pp. 054. doi:10.1088/1126-6708/2008/05/054

[20] J. P. Gauntlett and J. B. Gutowski, "Constraining Maximally Supersymmetric Membrane Actions,” Journal of High Energy Physics, Vol. 0806, 2008, p. 053. doi:10.1088/1126-6708/2008/06/053

[21] D. Gaiotto and E. Witten, “Janus Configurations, ChernSimons Couplings, and the Theta-Angle in $\mathrm{N}=4$ Super Yang-Mills Theory,” 2010. arXiv:0804.2907[hep-th]

[22] Y. Honma, S. Iso, Y. Sumitomo and S. Zhang, "Janus Field Theories from Multiple M2 Branes,” Physical Review D, Vol. 78, No. 7, 2008, Article ID: 025027, p 7. doi:10.1103/PhysRevD.78.025027

[23] K. Hosomichi, K.-M. Lee, S. Lee, S. Lee and J. Park, "N=5,6 Superconformal Chern-Simons Theories and M2Branes on Orbifolds," Journal of High Energy Physics, Vol. 0809, 2008, p. 002. doi:10.1088/1126-6708/2008/09/002

[24] M. Schnabl and Y. Tachikawa, "Classification of $N=6$ Superconformal Theories of ABJM Type,” 2008. arXiv:0807.1102[hep-th]

[25] M. A. Bandres, A. E. Lipstein and J. H. Schwarz, "GhostFree Superconformal Action for Multiple M2-Branes,” Journal of High Energy Physics, Vol. 0807, 2008, p. 117. doi:10.1088/1126-6708/2008/07/117

[26] P. de Medeiros, J. Figueroa-O’Farrill, E. Me’ndez-Escobar and P. Ritter, "On the Lie-Algebraic Origin of Metric 3-Algebras,” Communications in Mathematical Physics, Vol. 290, No. 3, 2009, pp. 871-902. doi:10.1007/s00220-009-0760-1
[27] S. A. Cherkis, V. Dotsenko and C. Saeman, "On Superspace Actions for Multiple M2-Branes, Metric 3-Algebras and Their Classification,” Physical Review D, Vol. 79, No. 8, 2009, Article ID: 086002, p 11. doi:10.1103/PhysRevD.79.086002

[28] P.-M. Ho, Y. Matsuo amd S. Shiba, "Lorentzian Lie (3-) Algebra and Toroidal Compactification of M/String Theory,” 2003. arXiv:0901.2003 [hep-th]

[29] K. Lee and J. Park, "Three-Algebra for Supermembrane and Two-Algebra for Superstring,” 2009. arXiv:0902.2417 [hep-th]

[30] P. de Medeiros, J. Figueroa-O’Farrill, E. Mendez-Escobar and P. Ritter, "Metric 3-Lie Algebras for Unitary Bagger-Lambert Theories," Journal of High Energy Physics, Vol. 0904, 2009, p. 037.

[31] C. Castro, "On n-Ary Algebras, Branes and Polyvector Gauge Theories in Noncommutative Clifford spaces," Journal of Physics A, Vol. 43, No. 36, 2010, Article ID: 365201. doi:10.1088/1751-8113/43/36/365201

[32] L. Smolin, "M Theory as a Matrix Extension of ChernSimons Theory," Nuclear Physics B, Vol. 591, No. 1, 2000, pp. 227-242. doi:10.1016/S0550-3213(00)00564-2

[33] L. Smolin, “The Cubic Matrix Model and a Duality between Strings and Loops,” 2000. hep-th/0006137

[34] T. Azuma, S. Iso, H. Kawai and Y. Ohwashi, "Supermatrix Models,” Nuclear Physics B, Vol. 610, No. 1, 2001, pp. 251-279. doi:10.1016/S0550-3213(01)00324-8

[35] N. Ishibashi, H. Kawai, Y. Kitazawa and A. Tsuchiya, “A Large-N Reduced Model as Superstring,” Nuclear Physics B, Vol. 498, No. 1, 1997, pp. 467-491. doi:10.1016/S0550-3213(97)00290-3

[36] B. de Wit, J. Hoppe and H. Nicolai, "On the Quantum Mechanics of Supermembranes,” Nuclear Physics B, Vol. 305, No. 4, 1988, pp. 545-581. doi:10.1016/0550-3213(88)90116-2

[37] B. Ezhuthachan, S. Mukhi and C. Papageorgakis, "D2 to D2,” Journal of High Energy Physics, Vol. 0807, 2008, p. 041.

[38] H. Singh, "SU(N) Membrane $B \wedge F$ Theory with DualPairs,” 2008. arXiv:0811.1690 [hep-th]

[39] K. Furuuchi and D. Tomino, "Supersymmetric Reduced Models with a Symmetry Based on Filippov Algebra," Journal of High Energy Physics, Vol. 0905, 2009, p. 070. doi:10.1088/1126-6708/2009/05/070

[40] H. Kawai and M. Sato, "Perturbative Vacua from IIB Matrix Model,” Physics Letters B, Vol. 659, No. 3, 2008, pp. 712-717. doi:10.1016/j.physletb.2007.11.021

[41] T. Eguchi and H. Kawai, "Reduction of Dynamical Degrees of Freedom in the Large N Gauge Theory," Physical Review Letters, Vol. 48, No. 16, 1982, pp. 1063-1066. doi:10.1103/PhysRevLett.48.1063

[42] G. Parisi, "A Simple Expression for Planar Field Theories,” Physics Letters B, Vol. 112, No. 6, 1982, pp. 463464. doi:10.1016/0370-2693(82)90849-8

[43] G. Bhanot, U. M. Heller and H. Neuberger, "The Quenched Eguchi-Kawai Model,” Physics Letters B, Vol. 113, No. 1, 1982, pp. 47-50. 


$$
\text { doi:10.1016/0370-2693(82)90106-X }
$$

[44] D. J. Gross and Y. Kitazawa, "A Quenched Momentum Prescription for Large N Theories," Nuclear Physics B, Vol. 206, No. 3, 1982, pp. 440-472. doi:10.1016/0550-3213(82)90278-4

[45] L. Motl, "Proposals on Nonperturbative Superstring Interactions,” 1997. hep-th/9701025

[46] T. Banks and N. Seiberg, "Strings from Matrices," Nuclear Physics B, Vol. 497, No. 1, 1997, pp. 41-55. doi:10.1016/S0550-3213(97)00278-2

[47] R. Dijkgraaf, E. Verlinde and H. Verlinde, "Matrix String Theory,” Nuclear Physics B, Vol. 500, No. 1, 1997, pp. 43-61. doi:10.1016/S0550-3213(97)00326-X
[48] J. M. Maldacena, "The Large N Limit of Superconformal Field Theories and Supergravity," Advances in Theoretical and Mathematical Physics, Vol. 2, No. 2, pp. 231252.

[49] M. Sato, "Model of M-theory with Eleven Matrices," Journal of High Energy Physics, Vol. 1007, 2010, p. 026. doi:10.1007/JHEP07(2010)026

[50] M. Sato, "Supersymmetry and the Discrete Light-Cone Quantization Limit of the Lie 3-Algebra Model of MTheory,” Physical Review D, Vol. 85, No. 4, 2012, Article ID: 046003, p 6. doi:10.1103/PhysRevD.85.046003

[51] M. Sato, "Zariski Quantization as Second Quantization," Physical Review D, Vol. 85, No. 12, 2012, Article ID: 126012, p 10. doi:10.1103/PhysRevD.85.126012 